\title{
Quality-assured solutions for green roof gardens on concrete deck with zero tolerance for leaks
}

\author{
Y. Edwards ${ }^{1}$, T. Emilsson ${ }^{2}$, J. Malmberg ${ }^{3}$, A. P Skog ${ }^{4}$ \\ \& C.-M. Capener \\ ${ }^{1}$ Swedish Cement and Concrete Research Institute, Stockholm, Sweden \\ ${ }^{2}$ Swedish University of Agricultural Sciences, Alnarp, Sweden \\ ${ }^{3}$ Scandinavian Green Roof Institute, Malmö, Sweden \\ ${ }^{4}$ Sweco Environment, Stockholm, Sweden \\ ${ }^{5}$ SP Technical Research Institute of Sweden, Borås, Sweden
}

\begin{abstract}
Eco-neighborhoods with gardens on concrete decks are for several reasons increasingly being prescribed today in major Swedish cities. However, there is a lack of knowledge, experience, standards and guidelines as well as collaboration between parties and stakeholders when installing such systems. It is incredibly important to avoid any leakage during the lifetime of a green roof garden but this cannot be completely guaranteed with today's installation practice and project management. At Sustainable City 2014 in Siena, we presented a paper about a new project aiming at bringing together researchers, government and industry to collaborative development of new and attractive solutions for green roof gardens with consideration to the environment and high requirements for durability, materials, construction and energy efficiency. This paper is a continuation of the paper presented in Siena and reports on the most recent results from the collaborative project which will finalize in November 2016. After that, the project will be further evaluated in a proposed continuation project for another couple of years.

Keywords: green roof garden, guidelines.
\end{abstract}




\section{Introduction}

A green roof installation on a building or other structure must be carefully built up from the concrete deck to be completely secured leak-proof. A water proofing system is generally placed on top of the concrete deck to prevent uncontrolled water from entering the building. The water proofing system normally includes a root barrier to prevent root penetration through the waterproofing layer and consequently also leakages. Thermal insulation is installed in combination with the waterproofing and root barrier. Finally, the vegetation and garden system visible to inhabitants and visitors is placed on top. This part of the construction is also based on a combination of several technical solutions including drainage layers, engineered soil/plant substrates, and geotextiles as means to ensure long term performance, integrity of the building envelope and successful delivery of ecosystem services such as:

- Contributing to a better stormwater management;

- Improving the proximity to recreation areas;

- Increasing biodiversity;

- $\quad$ Providing temperature reducing effects;

- $\quad$ Providing energy savings;

- Reducing noise;

- Providing cleaner air.

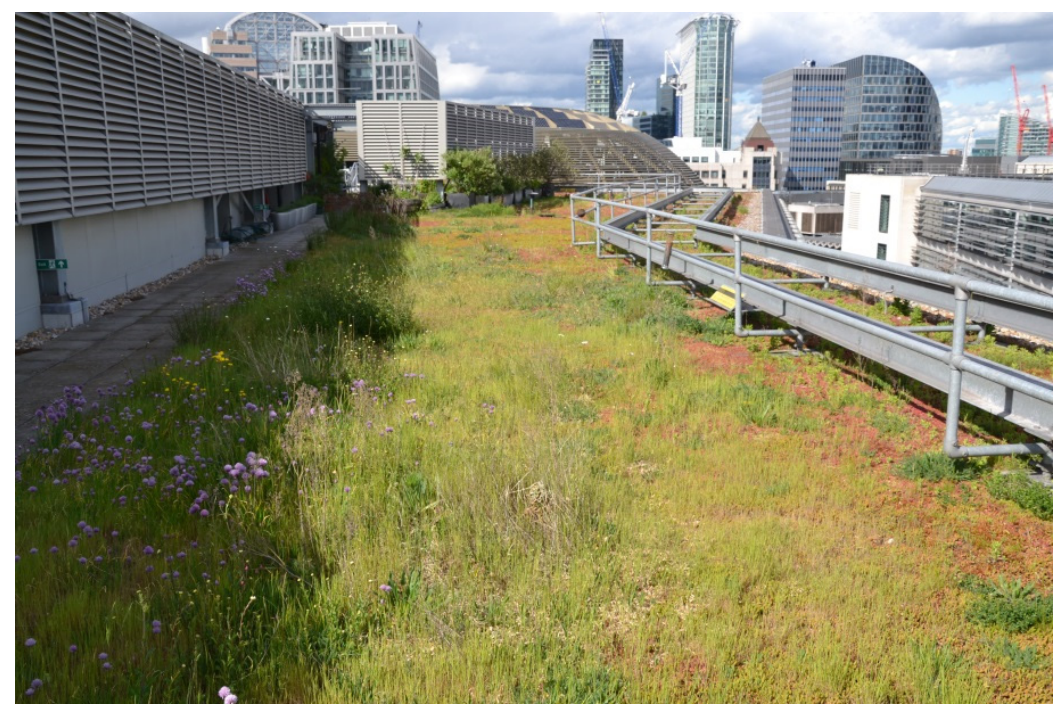

Figure 1: Climate-friendly green outdoor environment in London (photo: Ylva Edwards, CBI).

The goal of the project is to develop a network and forum, for developing new guidelines and certification system for Scandinavian conditions. This work will in turn lead to and result in durable and attractive construction solutions in the 
international forefront of green roof systems and similar facilities with measurable impact on sustainability in terms of durability, health, environment, economy, security and social interaction. At normal maintenance, these solutions will have to run smoothly for 100 years. Identifying gaps between different technologies involved in green roof construction is an essential part of the work.

The nineteen participating partners in the project come from the public sector, industry, architects and academia. They are all listed in [1] together with other participants in the project network.

Since 2013, the project is funded by Vinnova (Swedish Governmental Agency for Innovation Systems), is coordinated by the Swedish Cement and Concrete Research Institute and will finalize in November 2016 [2]. The project was presented at Sustainable City 2014 in Siena. Consequently, this paper is a continuation of the paper presented there.

More background to the project, involving its initial part (Stage 1) performed during 2013, is described in [1].

In this paper, work carried out so far within Stage 2 of the project is summarized.

\section{Project work: 2015}

Project design is shown in Figure 2.

For organizing the work, six work packages (WP) were created. These packages address:

- Problem analysis and knowledge inventory;

- Waterproofing system, insulation and concrete deck;

- Vegetation system and landscaping;

- Work process, from planning a green roof to maintenance;

- Standards, guidelines and certification;

- Coordination, administration, information, etc.

Work performed within three of the work packages listed above is briefly described in the following sections.

\subsection{WP - waterproofing system, insulation and concrete}

For roof waterproofing systems in green roof construction, resistance to root penetration, mechanical and chemical effects/degradation, high loads, stress and aging are required. Furthermore, the system must exhibit good flexibility and workability as well as being homogeneous and continuous under different types of vegetation. The choice of system depends on the type of roof (with or without thermal insulation in the waterproofing system, slope, etc.), load (use, service and maintenance, irrigation system, equipment, possible redevelopment of the building, etc.) and vegetation type (extensive or intensive).

The choice of thermal insulation also depends on type of roof, load, waterproofing system and vegetation type.

Regarding the concrete we have, in addition to the concrete substrate/roof as such, some other concrete installations or details to consider. These concern 


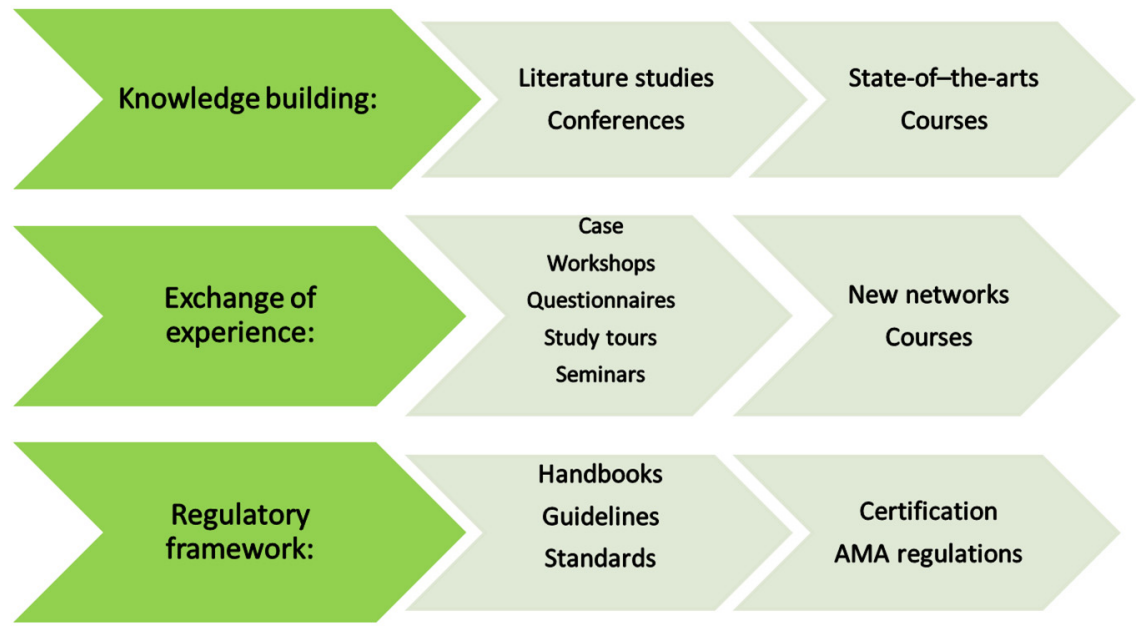

Figure 2: Design of project: Stage 2 .

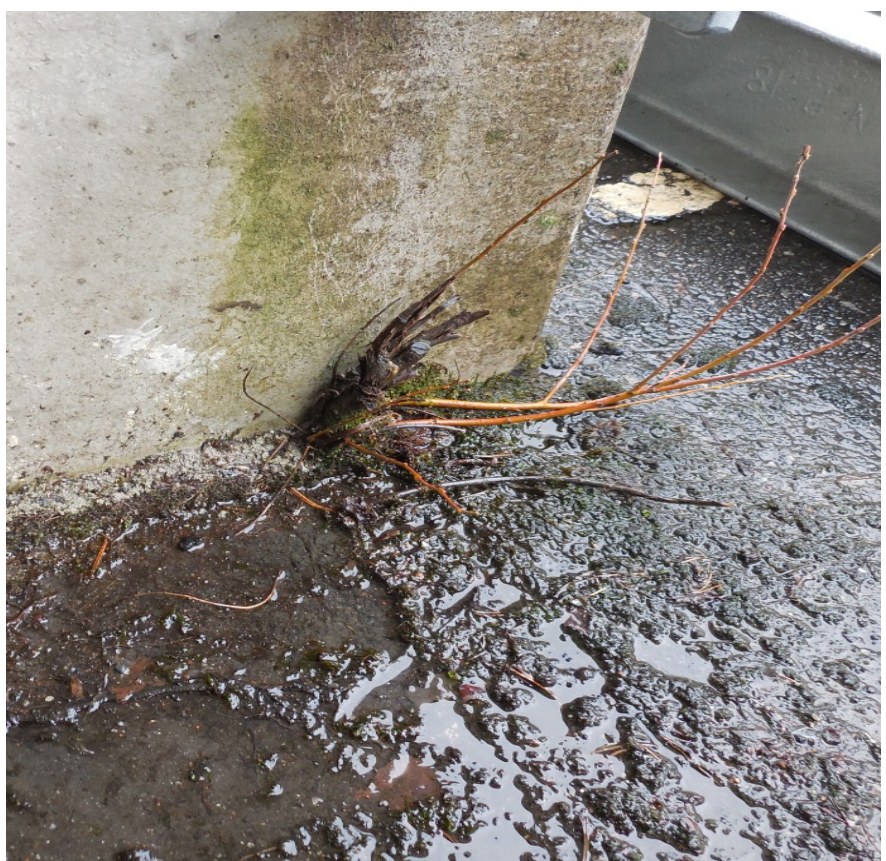

Figure 3: $\quad$ Concrete may need protection (photo: Dennis Lundgren, CBI).

protection concrete, screed, mortar and concrete for building up edges. Eventual problems concern for instance carbonates that can leach out and cause sintering in drainage facilities. Special treatment of such concrete installations may be needed. Obviously, using calcareous aggregates in drainage or protection layers is not 
suitable. So called waterproof concrete, without additional surface treatment /waterproofing system, is normally not considered an option for green roof installations in Sweden. Concrete products with high resistance to water are available on the market but, nevertheless, need protection of some kind.

The importance of a good concrete surface for the application of a waterproofing system cannot be overemphasized. The adhesion to concrete can never be better than its own surface tensile strength, and pretreatment work is therefore crucial. Pretreatment includes cleaning. All contaminants (such as dust, oil, grease and chemicals) must be removed as well as any laitance and curing membrane. This may be accomplished by grinding, milling and/or blasting. Vacuuming or water flushing may be necessary to get a really clean concrete surface with a good possibility for a primer product to penetrate the concrete.

The concrete surface must also be strong enough for the waterproofing coating, so that not any tensions that may arise at the interface between coating and concrete give rise to adhesion loss, and the coating thus becomes detached from the concrete. Surface tensile strength of the concrete shall be determined on site.

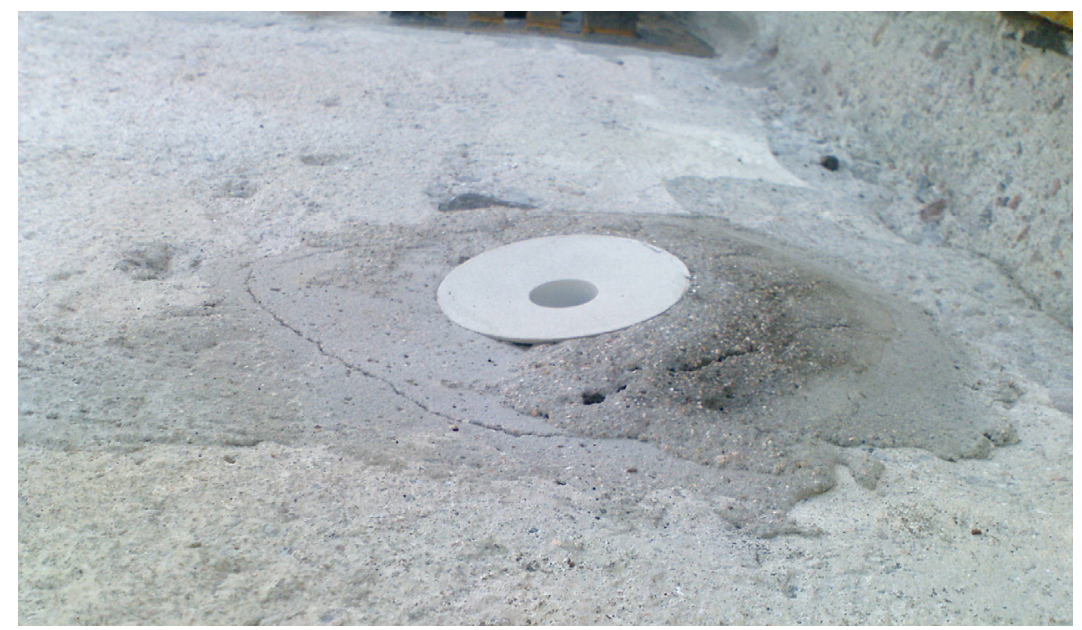

Figure 4: Concrete surface not suitable for installation of waterproofing (photo: Mikael Kinnmark, DAB Domiflex AB).

Surface temperature and moisture conditions are other important factors to consider for achieving good results. The surface temperature can often be crucial to the curing time of for instance a thermoset material.

Concrete shall normally always be primed/sealed before protection coating of any kind can be installed. The treatment is performed to increase adhesion between the concrete surface and coating. The primer is expected to penetrate into the concrete and wet the surface (low viscosity), have a certain moisture-resistant and dust-binding effects, and possibly also be able to reduce blistering in the application process. Primer and protective coating must be compatible and form a fully tested system depending on type of object and application. 
There are a number of different types of primer products for protective treatment of concrete, such as epoxy, urethane primer and acrylate based primer. Bitumen based primer products are used for bituminous waterproofing systems.

\subsubsection{Work performed}

Work carried out so far by members of the work package group dealing with waterproofing, insulation and concrete matters has resulted in a handbook covering these areas of a green roof installation. Material for the book has been prepared and discussed during numerous meetings organized as workshops by the work package leader in cooperation with other work package groups (see Chapter 2). The book includes a checklist for requirements as well as bad and good examples of green roof installations, concerning leakage, which have been identified and documented. This handbook will be available by the end of 2016 .

\subsection{WP - vegetation system and landscaping}

The current trend with increasing urban densities and transformation of ground based green spaces to systems on top of roof covered infrastructure or as roof gardens has led to a growing interest and need for building based vegetation systems with more interesting characters and more advanced plantations in relation to size of e.g. trees or delivery of particular ecosystem services. The traditional way of developing vegetation systems for roof gardens is by installing a drainage layer to allow discharge of excess water and a layer of soil substrate to anchor and support the vegetation with air, nutrients and water. Layers are most often separated by geotextile to maintain integrity of the materials. We see a need for deeper knowledge about substrate quality as well as the design and installation of different kinds of vegetation systems in order to achieve systems that can be described as sustainable. It includes knowledge about air and water holding capacity of different kinds of substrates and optimal depth for different performances. It also includes a description of feasible plant materials for different types of green roofs as well as determination of loads of vegetation and anchoring of trees at wind loads. In principal, we also see it important to have a thorough investigation of how different anthropocentric values as well as ecosystem service can be delivered by the use of different components of the vegetation systems as well as different design alternatives.

What is special about green roof installations is that the vegetation contact with groundwater is broken and that the entire water supply rests on the incoming rain or irrigation. This makes the plant bed particularly vulnerable during extreme weather events.

All types of green roof need a waterproofing as there will always be some runoff. Most if not all green roofs also need some form of drainage to channel away excess water during intense or extended rain events. The development of habitat or garden types opens up from almost unlimited choices, possibilities and complexity. We have identified substrate depth as the key driving character for most variables related to design but also to project cost as this puts different demands on supporting structures. 
In order to keep down the load on the concrete deck, haggling with the plant bed thickness is not uncommon. The consequence of doing so could be a system that does not support the vegetation properly due to deficient water storage for long drought periods as well as too low permeability causing low oxygen availability. Woody trees, shrubs and many perennials are particularly sensitive to lack of oxygen and the lower limit is considered to be around 10 percent by volume of air content. Limited water storage can be addressed by an irrigation system but this will at the same time exaggerate the anoxic conditions rapidly reducing vitality and survival of the vegetation.

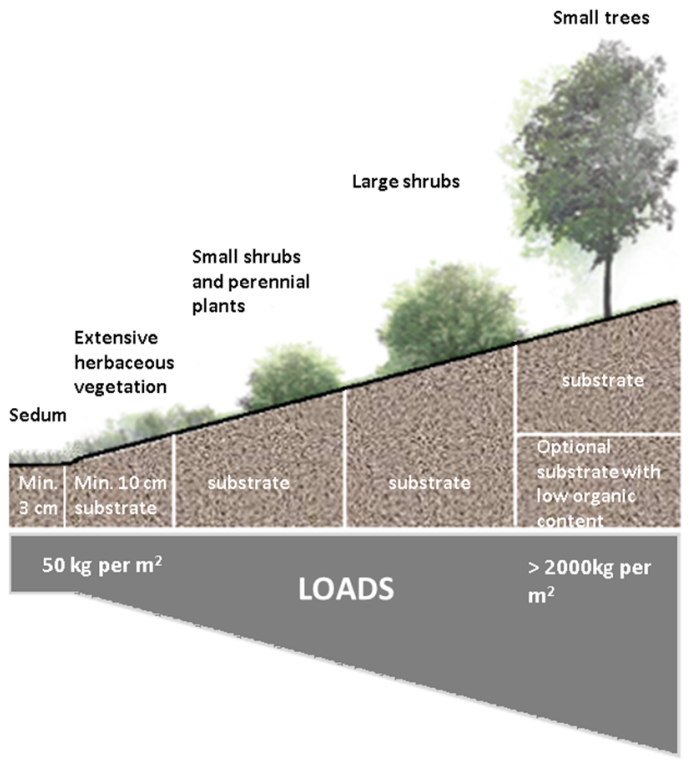

Figure 5: Different vegetation types require different installation depth, which in turn gives a different load on the supporting concrete deck.

\subsubsection{Work performed}

Work carried out so far by members of the work package group dealing with plant bed and vegetation has resulted in several articles, workshops and a handbook covering these areas of a green roof installation. Material has been prepared and discussed during numerous meetings organized by the work package leader in cooperation with other work package groups, in particular the work package group dealing with standards, guidelines and certification (see Chapter 2). The handbook will be available by the end of 2016 . 


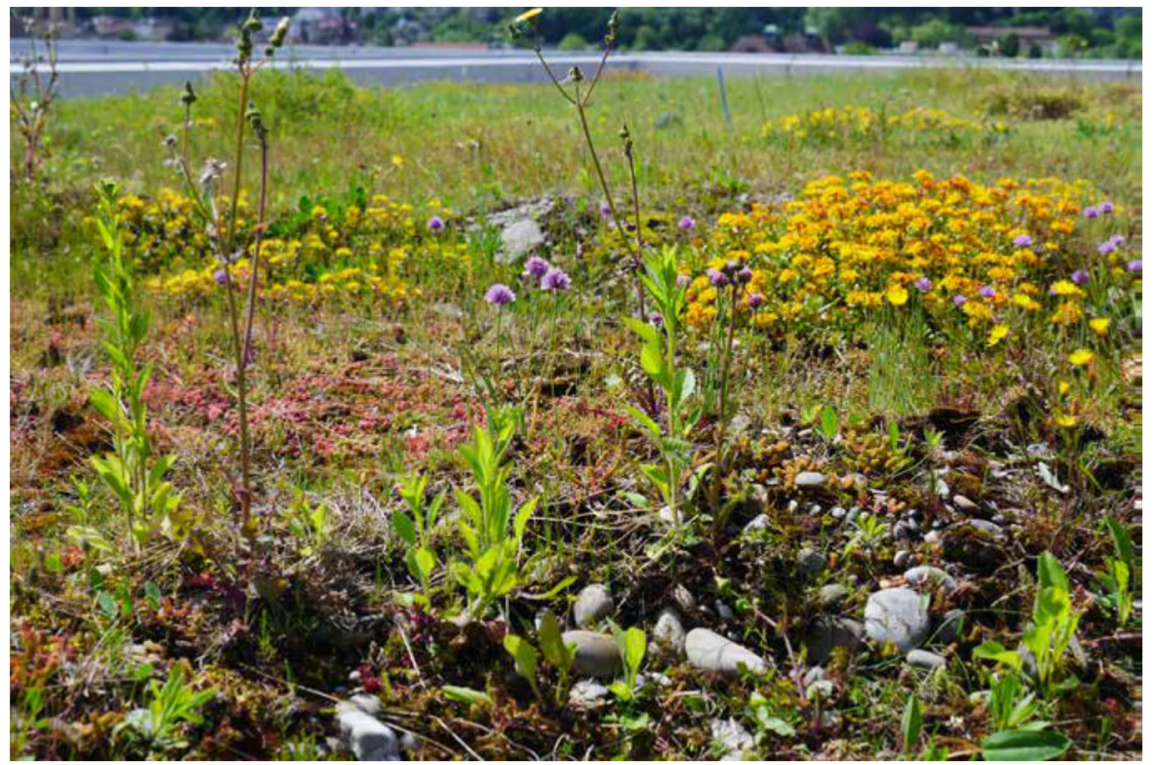

Figure 6: Examples of green roof with herbs, mosses and sedum vegetation. Substrate content and its depth (6-15) are varied over the entire surface of the roof to increase biodiversity (photo: Jonatan Malmberg, Scandinavian Green Roof Institute).

\subsection{WP - work process, from planning a green roof to maintenance}

In this work package, appropriate construction projects and buildings in Stockholm and Malmö are included for case studies. The selected four cases are Hornslandet, Hagastaden and Sergels torg in Stockholm and Greenhouse in Augustenborg, Malmö. Selection of construction projects started in late 2014. Selected cases are briefly described below.

Hornslandet is part of Norra Djurgårdsstaden, being one of Stockholm Environment profiled areas. The construction project includes some 150 apartments in houses with green roofs.

Hagastaden is a very complicated project carried out in various stages and involving many stakeholders in the project as a whole, such as Stockholm City, the Swedish Transport Administration and Stockholm Parkering (parking company). A small part of the Hagastaden project also forms part as a case in our project.

Our third case in Stockholm is Sergels torg. This plaza was built in the 60-70's. Now it leaks down through the roof of many shops located under the plaza, and has to be renovated. Joints are leaking the most. Although this case is not really a roof garden case, except for trees, it was included in our project because of interesting waterproofing membrane solutions. The Traffic Office and Stockholm City are the promoters of the project and Skanska is the project contractor. 
The greenhouse construction project consists of three interconnected units: one fourteen-story building with about 34 apartments, twelve terraced houses and one eco-profile preschool building. On the roof of the fourteen-story building, there are extensive green roof plantations and on top of the preschool building there is a rooftop garden for urban community gardening and a large greenhouse. Furthermore, each of the apartments in the fourteen-story building have large specially designed and reinforced balconies of 22 square meters where one can grow vegetables and other plants. Estimated completion is in spring 2016. The construction project is built by MKB, a real estate company owned by Malmö City in partnering with NCC.

For all four cases: input, analysis, evaluation, documentation and follow-up of the entire green roof construction system are being delivered to our project.

\subsubsection{Work performed}

Work carried out so far by members of the work package group dealing with the work process has involved numerous interviews and reviews of planning documents. Interview questions were divided into main categories concerning requirements, handovers and monitoring/inspections. The interviews were conducted with project managers, project leaders and site managers. A query matrix was developed to support the interviews. The outcome of interviews and group discussions and ideas from meetings and workshops are summarized in a report whose main purpose is to provide input to the work package dealing with the development of guidelines. A number of shortcomings have been identified in the work process of green roof constructions.
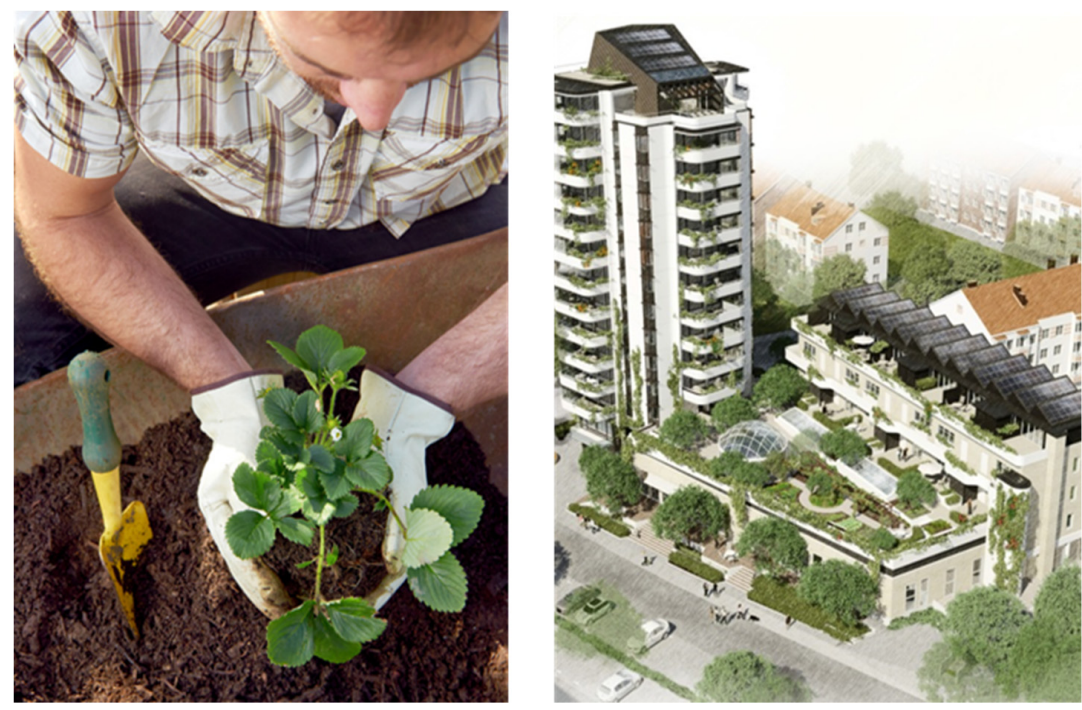

Figure 7: Greenhouse in Augustenborg, Malmö with cultivation on balconies. 


\section{Conclusions and work in progress}

The project is focusing on comprehensive solutions in the light of all input from the initialization process carried out in 2013. Current standards and guidelines used for green roof installations have been analyzed and sorted into distinct areas of knowledge and experience. Work carried out within the different work packages have resulted in:

- Workshops, seminars and courses;

- Articles and input to regulations;

- Expanded network;

- Handbook on the selection of materials for waterproofing, insulation and concrete;

- Handbook for the selection of plant bed and vegetation;

- Report based on interviews and reviews of planning documents linked to four on going big construction projects in Stockholm and Malmö. The report will give valuable input to the work carried out on green roof guidelines for the future.

\section{References}

[1] Y. Edwards, T. Emilsson, J. Malmberg, A. P. Skog, Quality-assured Solutions For Green Roof Gardens On Concrete Decks With Zero Tolerance For Leaks, The Sustainable City IX (2 Volume Set), 2014.

[2] Vinnova, www.vinnova.se 